\title{
Strong influence of solar X-ray flares on low-frequency electromagnetic signals in middle latitudes
}

\author{
Alexander Rozhnoi ${ }^{1}$, Maria Solovieva ${ }^{1}$, Viktor Fedun ${ }^{2}$, Peter Gallagher ${ }^{3}$, Joseph McCauley ${ }^{3}$, \\ Mohammed Y. Boudjada ${ }^{4}$, Sergiy Shelyag ${ }^{5}$, and Hans U. Eichelberger ${ }^{4}$ \\ ${ }^{1}$ Schmidt Institute of Physics of the Earth of RAS, 10-1 B. Gruzinskaya St., 123242 Moscow, Russia \\ ${ }^{2}$ Plasma Dynamics Group, Department of Automatic Control and Systems Engineering, \\ University of Sheffield, Mappin 6 St., Sheffield, S1 3JD, UK \\ ${ }^{3}$ Trinity College Dublin, the University of Dublin, Dublin 2, Ireland \\ ${ }^{4}$ Space Research Institute of AAS, Schmiedlstraße 6, 8042 Graz, Austria \\ ${ }^{5}$ School of Information Technology, Deakin University, Geelong, Australia
}

Correspondence: Viktor Fedun (v.fedun@sheffield.ac.uk)

Received: 1 April 2019 - Discussion started: 17 April 2019 Accepted: 26 August 2019 - Published: 23 September 2019

\begin{abstract}
In this paper we analysed sudden phase anomalies (SPAs) of VLF-LF signals recorded at Graz (Austria), Birr (Ireland) and Moscow (Russia) stations during two strong solar flares in September 2017. The first X-class 9.3 flare occurred on 6 September at 12:02 UT, and the second X-class 8.2 flare was observed on 10 September 2017 at 16:06 UT. Data from seven transmitters in a frequency range between 20 and $45 \mathrm{kHz}$ are used for the analysis. The SPAs were observed in all middle-latitude paths (differently orientated) with path lengths from 350 to $7000 \mathrm{~km}$. Solar X-ray burst data were taken from GOES satellite observations in the wavelength band of $0.05-0.4 \mathrm{~nm}$. It was found that (i) the amplitude of SPAs in different paths varies from 10 to $282^{\circ}$, and (ii) the correlation between the amplitudes of SPAs, the lengths of paths and the signal frequency is weak. The change in effective height of reflection due to lowering of the reflecting layer during the flares was found to be about $12 \mathrm{~km}$ for the first event and about $9 \mathrm{~km}$ for the second event. Spectral analysis of the X-ray and LF data, filtered in the range between $5 \mathrm{~s}$ and $16 \mathrm{~min}$, showed that the LF signal spectra are very similar to X-ray spectra. Maxima of both X-ray and LF spectra are in 2-16 min interval.
\end{abstract}

\section{Introduction}

Solar flares are one of the most influential space weather events. During the flares, electromagnetic and corpuscular radiation of significant power is emitted. Arrival of the solar proton radiation is mainly observed in the regions with magnetic latitudes greater than $60^{\circ}$. The protons produce polar cap absorption events, which can last up to several days. In the middle latitudes, significant changes in the lower ionosphere electron density are induced by solar X-ray flares.

During most of the solar X-ray flares the electron density is strongly enhanced due to sudden increases in extreme ultraviolet and hard X-ray radiation. Ionisation is usually produced below the normal D region (e.g. Chilton et al., 1963; Whitten and Poppoff, 1965), and this region responds dramatically to the X-ray flares. Although the main source of ionisation, Lyman- $\alpha$ emission, is enhanced during the flare event, the X-ray emission overwhelms its effect several times, leading to 1-2 orders of magnitude increase in the Dregion electron density (e.g. Grubor et al., 2008).

Very low and low frequency (VLF-LF) radio wave measurements are used to study the D-region behaviour. These waves are reflected by the $\mathrm{D}$ region, and changes in ionisation of the $\mathrm{D}$ region result in amplitude and phase variations of the received VLF signal. Since Kreplin et al. (1962) have reported the relationship between X-ray bursts during flares and sudden phase anomalies (SPAs), numerous works have been published on the studies of flare characteristics 
and SPA phenomenology (e.g. Mitra, 1974; Muraoka et al., 1977; Kamada, 1985; Khan et al., 2005). Many authors have studied the ionospheric response to solar flares in the last few decades (e.g. Žigman et al., 2007; Druzhin et al., 2014; Hayes et al., 2017). Works of Raulin et al. (2010, 2013) used data from the South American VLF Network (SAVNET) to study a large number of events in order to determine the Xray threshold, which produces ionospheric disturbances. Recently, Li et al. (2019) studied the ionospheric response to solar flares based on medium-frequency (MF) radar and found that the electron density $\left(N_{\mathrm{e}}\right)$ and effective reflection height $(H)$ had profound responses to solar flares. Liu et al. (2018) investigated the ionospheric D layer height fluctuations, during the solar flares, by analysing the VLF-LF waveforms emitted by lightning. It was found that the short-time fluctuations of D layer height are linearly correlated to the flux density of solar radiations.

The boundary between the ionospheric $\mathrm{D}$ region and the atmosphere is characterised by two parameters: sharpness $(\beta$ in $\mathrm{km}^{-1}$ ) and reflection height $\left(H^{\prime}\right.$ in $\left.\mathrm{km}\right)$. For unperturbed daytime ionosphere, $\beta=0.3$ and $H^{\prime}=71 \mathrm{~km}$ (Wait and Spices, 1964). During an X-ray flare, the sunlit ionosphere reflection height decreases considerably, while the sharpness increases in comparison with the unperturbed ionosphere. Thomson et al. (2005) modelled the D-region electron densities as functions of X-ray flux up to the level of the largest, X45 flare. During this largest flare, they found that the reflecting height was about $17 \mathrm{~km}$ below the normal midday level. Grubor et al. (2008) investigated changes in the ionosphere during X-ray flares (ranging from class $\mathrm{C}$ to $\mathrm{M}$ ), which have the corresponding VLF recordings from Belgrade in 2004-2007. They found the decrease in ionosphere reflection height of up to $7 \mathrm{~km}$ and $\beta=0.47$ during the strongest events.

The study of ionospheric response to solar flares remains important research, considering the possibility of improvements in the capacity and reliability of predicting disturbances in space weather, which can influence quality of radio communications. The strongest effect on radio wave propagation comes from the daytime low ionospheric variations. They can produce disruptions in radio communication and cause problems with navigation.

In this work we investigate SPA characteristics from the VLF-LF signals recorded during two strong solar flares in September 2017 in the three European middle-latitude VLF stations - Graz (Austria), Birr (Ireland) and Moscow (Russia). Our VLF-LF receiving UltraMSK (http://ultramsk.com, last access: 13 August 2019) stations were developed in New Zealand and simultaneously record both the amplitude and phase of MSK (minimum-shift keying) modulated signals in the frequency range $10-50 \mathrm{kHz}$ from several VLF-LF transmitters. MSK signals have fixed frequencies in narrow band $50-100 \mathrm{~Hz}$ around the main frequency and adequate phase stability. The receiver station consists of the VLF antenna with pre-amplifier, GPS receiver with GPS antenna for accu- rate signal timing, analog-to-digital converter and computer with special software. The receiver can record signals with time resolutions ranging from $50 \mathrm{~ms}$ to $60 \mathrm{~s}$. For our purpose we use sampling rate of $20 \mathrm{~s}$. The receivers work continuously (e.g. the receiver in Kamchatka has been working continuously since 2000).

Although the sudden ionospheric disturbances from solar flares on the VLF signal are always well recognisable, they can vary substantially along the different way paths. The series of powerful solar flares started on 4 September from geoeffective Active Region 2673. The biggest flare to erupt from this active sunspot occurred on 6 September. According to the data from the geostationary satellite GOES 15 (https: //www.swpc.noaa.gov/, last access: 13 September 2019), the strongest solar flare started at 11:53 on 6 September 2017, peaked as X9.3 at 12:02 UT and ended at 12:10 UT. This was the second X-class solar flare of that day. It came just a few hours after a long-duration X2.2 at 09:33 UT.

The second strong solar flare from the same active region was recorded on 10 September. The event began at 15:35 achieving the maximum at 16:06 as X8.2 and ended at 16:31 UT.

\section{Results of data analysis}

Data from the eight ground-based transmitters in the frequency range from about 20 to $45 \mathrm{kHz}$ were used for the analysis (Fig. 1). The SPAs were observed in the middle-latitude paths with lengths from 350 to $6000 \mathrm{~km}$ and different orientations. A typical SPA is characterised by a sudden advance of phase followed by a rounded maximum and, generally, an exponential decay or a recovery phase. Sometimes SPAs have gradual onset. Infrequently, events have either a complex onset or complex phase variation. The classification of events as simple (S), gradual (G) and complex (C) types of SPAs was introduced by Kamada (1985). Two examples (together with amplitude variations) are shown in Figs. 2 and 3.

SPA characteristics for 6 September 2017 and for 10 September 2017 are shown in Tables 1 and 2, respectively. In total, 15 wave paths were analysed for 6 September, and 17 wave paths were analysed for 10 September events. The amplitude and phase anomalies due to the solar flares were observed in all the paths. The majority of anomalies on 6 September had a simple form (an exception is the path GBZ-GRZ, which had a complex onset). All of the anomalies were positive, and the shapes of SPA dependencies on time closely resemble those for X-ray irradiation. The onset time (the time difference between the beginning of the flare and the start of SPA) was about $4 \mathrm{~min}$ for most of the events. This is in a good agreement with Deshpande et al. (1972), who found a delay of 3-4 min between the beginnings of Xray and SPA events. The SPA duration was on average about $26 \mathrm{~min}$. The period of recovery was about $1 \mathrm{~h}$. 


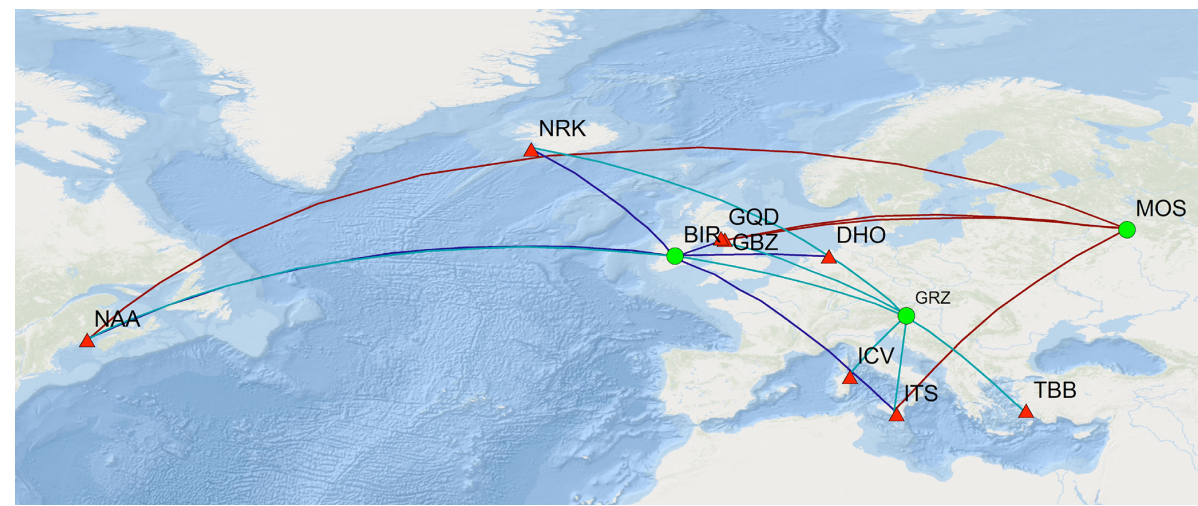

Figure 1. Positions of the receivers (green circles) in Birr, Ireland (BIR), Graz, Austria (GRZ) and Moscow, Russia (MOS) together with positions of the eight transmitters (red triangles) that have been used in this study.

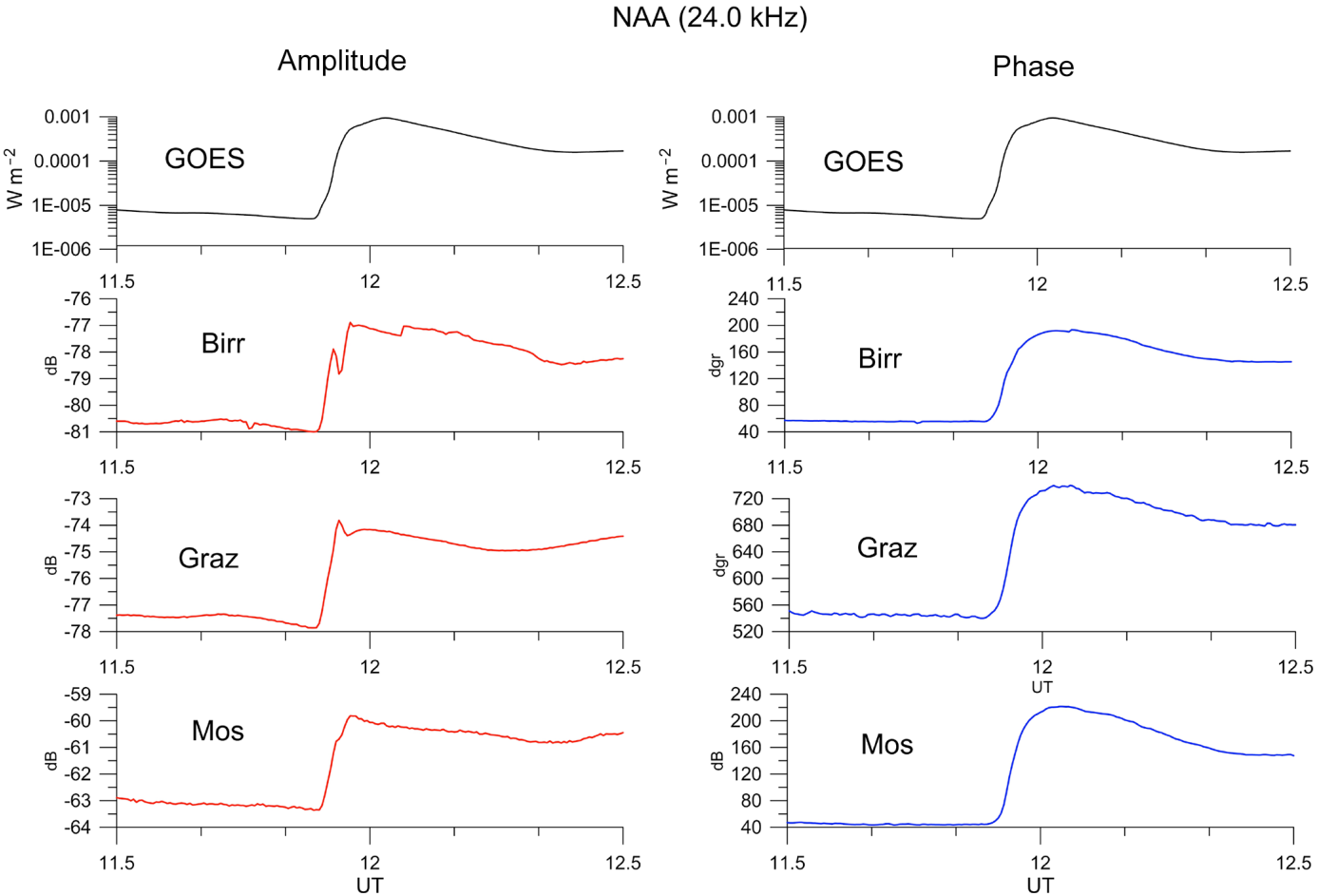

Figure 2. Amplitude (left) and phase (right) anomalies recorded in the NAA signal in Birr, Graz and Moscow receiving stations during the X-ray burst on 6 September 2017. Top panel shows X-ray flux measured with GOES satellite in the wavelength range of $0.05-0.4 \mathrm{~nm}$.

The SPAs on 10 September were more gradual due to the longer flare growth. All the SPAs on 10 September were positive, and their duration was between 30 and $60 \mathrm{~min}$. The recovery period for this day is difficult to estimate because the flare occurred close to the evening terminator.

The majority of the amplitude anomalies of VLF-LF signals were positive except for six paths (an example of negative anomaly is shown in Fig. 3).

The amplitudes of SPAs in different paths vary from 10 to $282^{\circ}$. A weak correlation between the amplitudes of SPAs and the ratio of the path lengths to the signal wavelength was found (see Fig. 4). The change in the effective height of reflection $(\Delta h)$ due to the lowering of the reflecting layer during a flare has been calculated using the formulas of Westfall (1961) for the paths longer than $1000 \mathrm{~km}$ and large flares.

$$
\Delta h=-\frac{c \Delta t}{d\left(\frac{\lambda^{2}}{16 h^{3}}+\frac{1}{2 a}\right)}\left[1+\frac{3 \lambda^{2} c \Delta t}{32 \lambda^{4} d\left(\frac{\lambda^{2}}{16 h^{3}}+\frac{1}{2 a}\right)^{2}}\right],
$$

where $d$ is the distance between transmitter and receiver in $\mathrm{km}, \lambda$ is the wavelength of signal in $\mathrm{km}, a$ is the radius of the Earth, $c$ is the velocity of light, $\Delta t$ is the phase shift, $h$ is the 
GBZ (19.58 kHz)

Amplitude
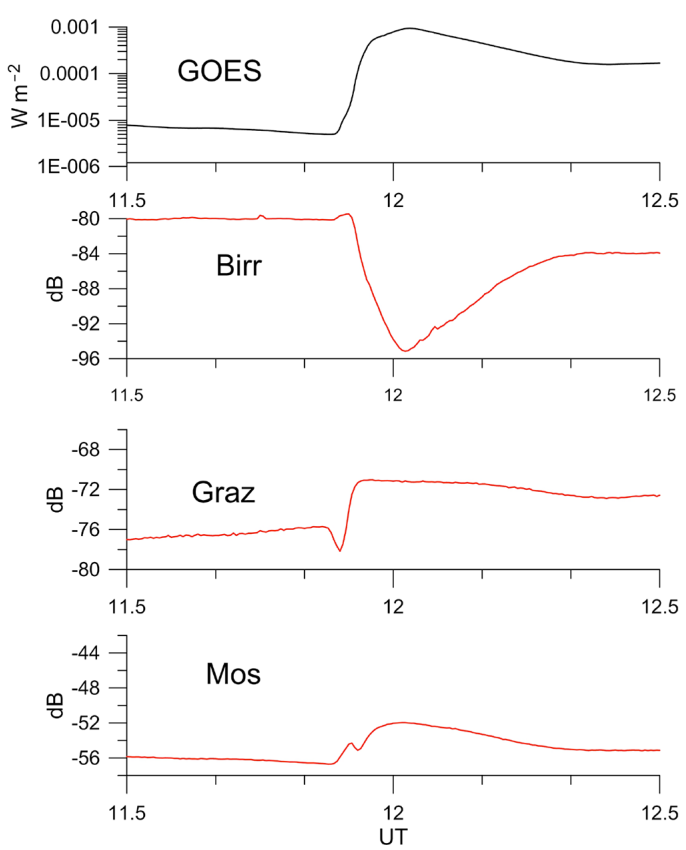
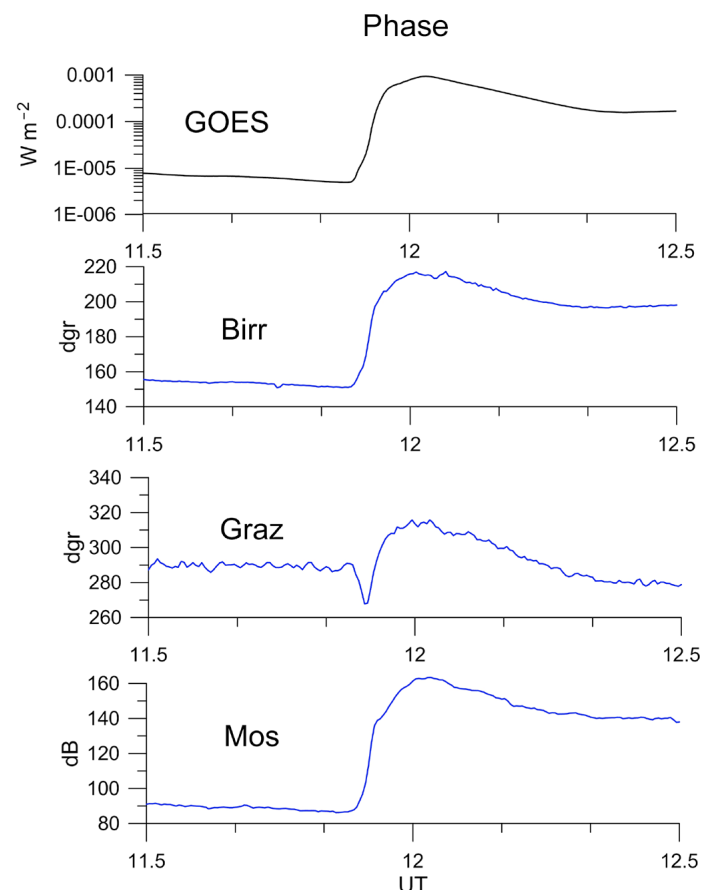

Figure 3. Same as Fig. 2 but for the signal from GBZ transmitting station.

Table 1. SPAs in VLF-LF signals observed on 6 September 2017.

\begin{tabular}{lrrrrrr}
\hline Path & Transmitter power, W & Frequency, $\mathrm{kHz}$ & Length, km & Orientation & $A_{\text {max }}{ }^{\circ}$ & Duration, min \\
\hline NAA-GRZ & 1000 & 24.0 & 6000 & $\mathrm{~W}-\mathrm{E}$ & 196 & 26 \\
NAA-BIR & 1000 & 24.0 & 4300 & $\mathrm{~W}-\mathrm{E}$ & 137 & 25 \\
NAA-MOS & 1000 & 24.0 & 7000 & $\mathrm{~W}-\mathrm{E}$ & 175 & 27 \\
NRK-GRZ & 100 & 37.5 & 3000 & $\mathrm{~N}-\mathrm{S}$ & 62 & 25 \\
NRK-BIR & 100 & 37.5 & 1500 & $\mathrm{~N}-\mathrm{S}$ & 128 & 28 \\
GBZ-GRZ & 30 & 19.58 & 1500 & $\mathrm{~N}-\mathrm{S}$ & 26 & 22 \\
GBZ-BIR & 30 & 19.58 & 350 & $\mathrm{E}-\mathrm{W}$ & 65 & 23 \\
GBZ-MOS & 30 & 19.58 & 2500 & $\mathrm{~W}-\mathrm{E}$ & 80 & 27 \\
ICV-GRZ & 20 & 20.27 & 850 & $\mathrm{~S}-\mathrm{N}$ & 70 & 27 \\
ITS-GRZ & - & 45.9 & 1100 & $\mathrm{~S}-\mathrm{N}$ & 157 & 26 \\
ITS-MOS & - & 45.9 & 2700 & $\mathrm{~W}-\mathrm{E}$ & 177 & 26 \\
ITS-BIR & - & 45.9 & 2500 & $\mathrm{~S}-\mathrm{N}$ & 165 & 24 \\
TBB-GRZ & - & 26.7 & 1500 & $\mathrm{~S}-\mathrm{N}$ & 106 & 27 \\
GQD-BIR & 60 & 22.1 & 350 & $\mathrm{E}-\mathrm{W}$ & 54 & 27 \\
GQD-MOS & 60 & 22.1 & 2500 & $\mathrm{~W}-\mathrm{E}$ & 75 & 27 \\
\hline
\end{tabular}

reflection height, and $\Delta h$ is the decrease in reflection height. It was found that $\Delta h$ varies considerably from about $2 \mathrm{~km}$ (GBZ-GRZ) to 20-22 km (e.g. NAA-GRZ, NRK-GRZ). On average, the lowering of the reflecting layer was about $12 \mathrm{~km}$ for the first event and about $9 \mathrm{~km}$ for the second event. In $75 \%$ of the case the decrease in the height of the reflecting layer was more than $7 \mathrm{~km}$.

To establish, based on the data, a statistical relation between the additional X-ray input onto the lower ionosphere and the variations seen in the VLF paths we calculated the following parameters: X-ray flux vs. VLF phase and X-ray flux vs. VLF amplitude. Both VLF phase and amplitude were scaled with the zenith angle of the receiving station. Examples for 6 September at the Birr station are shown in Fig. 5.

All graphs show good correlation between the X-ray flux and VLF signals; however, the relationship for the phase is more stable. The amplitude for the path GBZ-BIR has inclination to the left because the amplitude anomaly was neg- 
Table 2. SPAs in VLF-LF signals observed on 10 September 2017.

\begin{tabular}{|c|c|c|c|c|c|c|}
\hline Path & Transmitter power, W & Frequency, kHz & Length, km & Orientation & $A_{\max },{ }^{\circ}$ & Duration, min \\
\hline NAA-GRZ & 1000 & 24.0 & 6000 & W-E & 149 & 52 \\
\hline NAA-BIR & 1000 & 24.0 & 4300 & W-E & 122 & 45 \\
\hline NAA-MOS & 1000 & 24.0 & 7000 & W-E & 120 & 50 \\
\hline NRK-GRZ & 100 & 37.5 & 3000 & $\mathrm{~N}-\mathrm{S}$ & 282 & 63 \\
\hline NRK-BIR & 100 & 37.5 & 1500 & $\mathrm{~N}-\mathrm{S}$ & 76 & 64 \\
\hline GBZ-GRZ & 30 & 19.58 & 1500 & $\mathrm{~N}-\mathrm{S}$ & 20 & 50 \\
\hline GBZ-BIR & 30 & 19.58 & 350 & $\mathrm{E}-\mathrm{W}$ & 55 & 66 \\
\hline GBZ-MOS & 30 & 19.58 & 2500 & W-E & 27 & 60 \\
\hline ICV-GRZ & 20 & 20.27 & 850 & $\mathrm{~S}-\mathrm{N}$ & 47 & 50 \\
\hline ITS-GRZ & - & 45.9 & 1100 & $\mathrm{~S}-\mathrm{N}$ & 98 & 54 \\
\hline ITS-MOS & - & 45.9 & 2700 & W-E & 100 & 45 \\
\hline TBB-GRZ & - & 26.7 & 1500 & $\mathrm{~S}-\mathrm{N}$ & 70 & 52 \\
\hline GQD-BIR & 60 & 22.1 & 350 & E-W & 50 & 45 \\
\hline GQD-MOS & 60 & 22.1 & 2500 & W-E & 52 & 47 \\
\hline DHO-GRZ & 800 & 23.4 & 900 & $\mathrm{~N}-\mathrm{S}$ & 43 & 38 \\
\hline DHO-BIR & 800 & 23.4 & 1000 & E-W & 10 & 24 \\
\hline DHO-MOS & 800 & 23.4 & 2000 & W-E & 50 & 12 \\
\hline
\end{tabular}

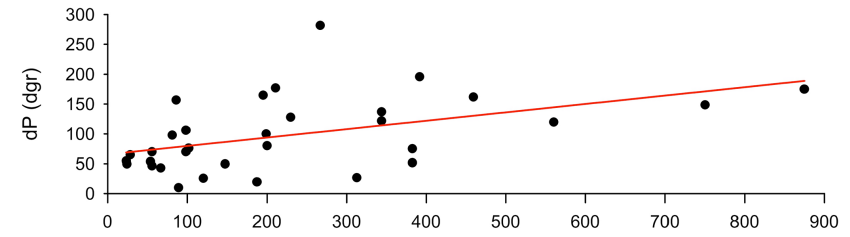

Figure 4. The plot of SPAs against $d / \lambda$ ( $d$ is distance in $\mathrm{km}, \lambda$ is wavelength in $\mathrm{km}$ ).

ative (see Fig. 3). The NAA amplitude anomaly was not smooth (see Fig. 2), and therefore its graph is distorted.

\subsection{Spectral analysis}

The data from GOES satellite and the phase data from the wave path NRK-BIR were used for the spectral analysis. The X-ray GOES data with the wavelength of $0.05-0.4 \mathrm{~nm}$ and the temporal resolution of $2 \mathrm{~s}$ were filtered in the range of $5 \mathrm{~s}-16 \mathrm{~min}$. The NRK-BIR path phase data have the cadence of $1 \mathrm{~s}$. The acquired spectra are shown in Fig. 6. For comparison, the spectra of 4 undisturbed days are provided in Fig. 6c. The spectra of the LF signals are very similar to the spectra of X-ray measurements. Maxima for both X-ray and LF spectra are in the interval of $2-16 \mathrm{~min}$. The spectra rapidly decrease with fluctuations less than $2 \mathrm{~min}$. The spectra for undisturbed days have the maxima of about 3-4 min, and their decrease is insignificant.
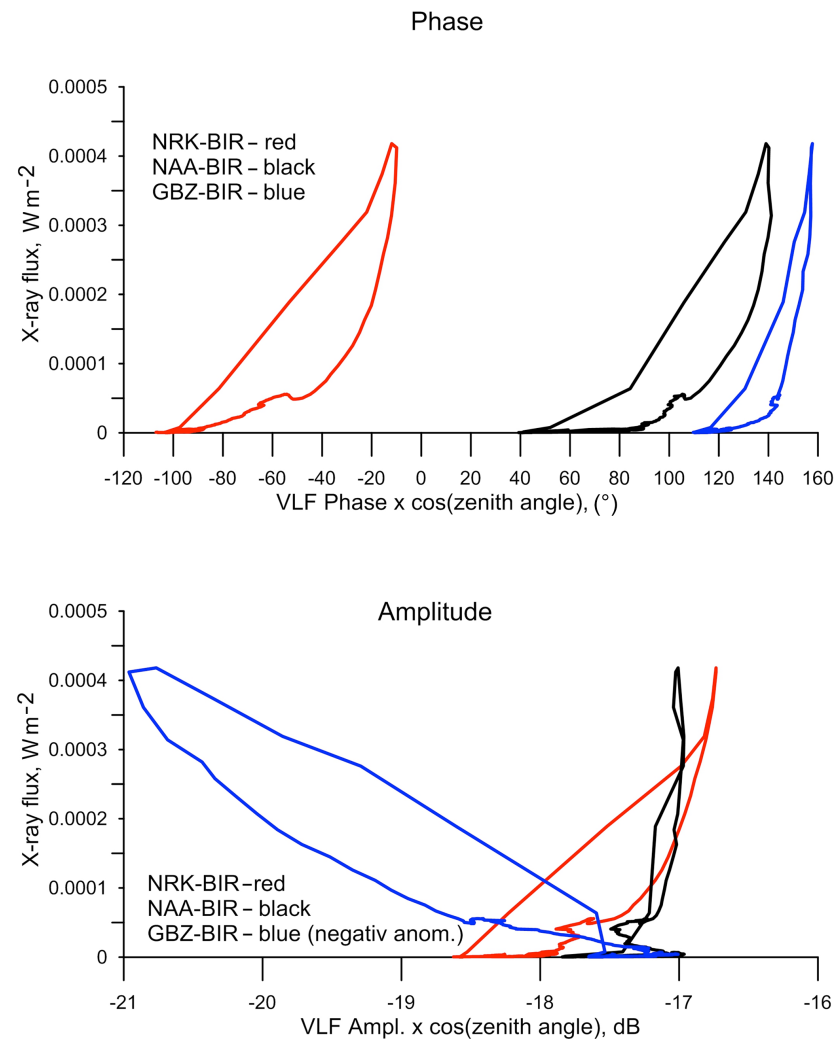

Figure 5. X-ray flux (irradiance) vs. VLF phase and amplitude scaled with the zenith angle of the receiving station in Birr. 


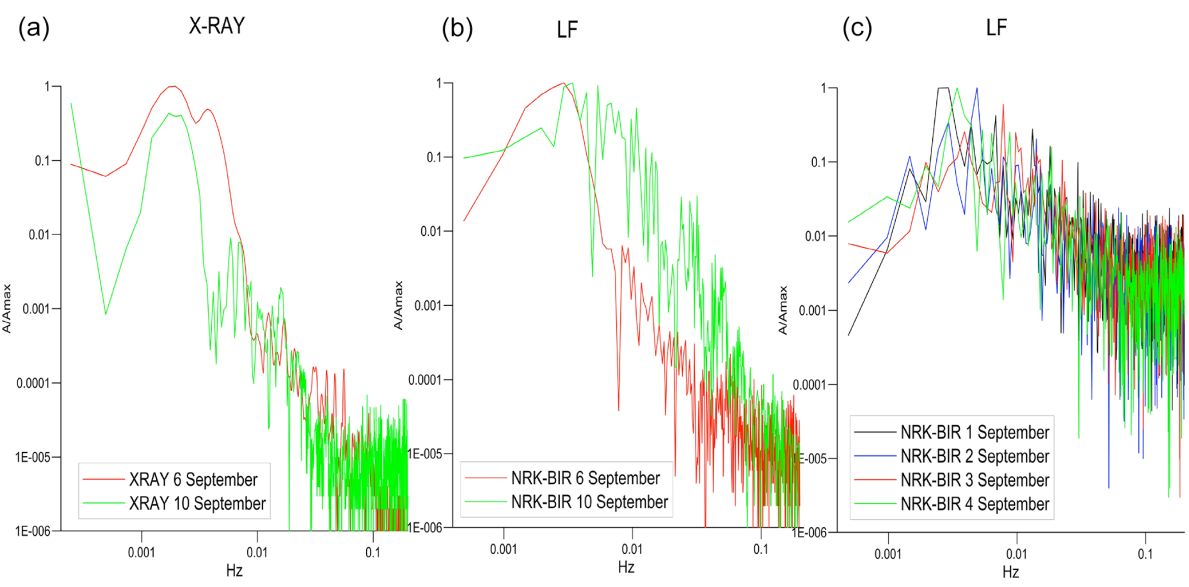

Figure 6. Spectra of X-ray (a) and LF (b) data (filtered in range $5 \mathrm{~s}-16 \mathrm{~min}$ ) for the two solar flares. The spectra of the same signal for quiet days are shown in panel (c) for comparison.
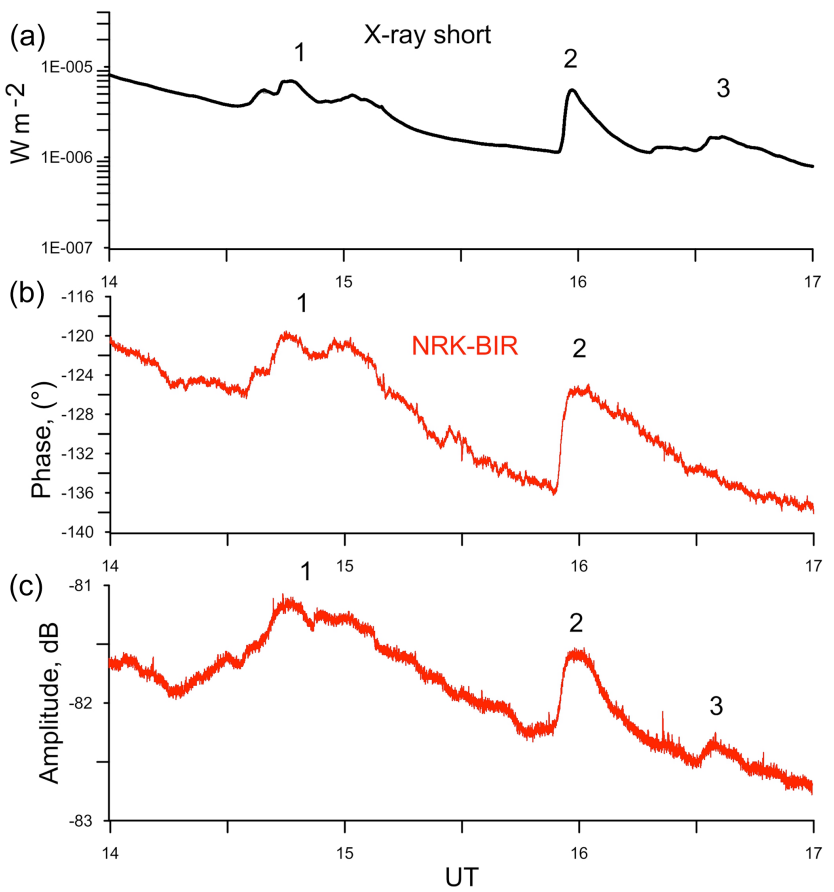

Figure 7. Fluctuations of X-ray flux for X9.3 solar flare on 6 September during the recovery phase (a) and phase (b) and amplitude (c) variations of the signal in the NRK-BIR path (temporal resolution is $1 \mathrm{~s})$.

\subsection{Response of the $D$ region of the ionosphere to weak (class C) X-ray fluctuations during the recovery phase}

Figures 7 and 8 show the influence of the weak X-ray fluctuations on the lower ionosphere. The influence can be clearly seen in the received VLF-LF amplitude and phase of the NAA and NRK signals recorded in BIR station on 6 September. The resolution of VLF-LF data in this case was $1 \mathrm{~s}$.

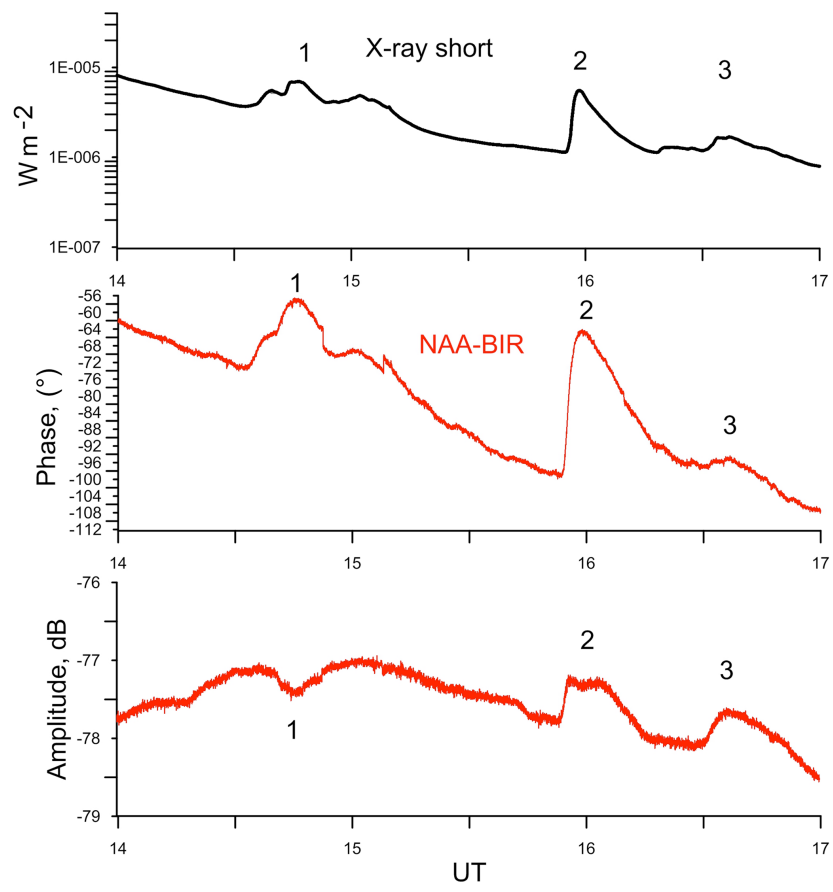

Figure 8. Same as in Fig. 7 for the wave path NAA-BIR.

It can be seen that VLF-LF signals repeat variations of Xray signals. This fact is in agreement with the detection of solar flare pulsations in the Earth's ionosphere (e.g. Hayes et al., 2017). Authors made the first observations of synchronised quasi-periodic pulsations in X-ray flaring emission (class $\mathrm{C}$ ) in ionospheric $\mathrm{D}$ region. The $\mathrm{D}$-region response was observed using VLF $(24 \mathrm{kHz})$ signal. Variations of the VLF signal repeat the quasi-periodic pulsations in X-ray (recorded in satellite the GOES) with delay of $90 \mathrm{~s}$. 


\section{Conclusions}

In this study, we investigated sudden phase anomalies (SPAs) in VLF-LF signals (frequency range $20-45 \mathrm{kHz}$ ) propagating in the middle-latitude paths during two solar flares with intensities X9.3 and X8.2 in September 2017. Path lengths varied from 350 to $6000 \mathrm{~km}$, and their orientation was different. In total 15 wave paths were analysed on 6 September, and 17 paths were analysed on 10 September. Our main findings are as follows:

- Amplitude and phase anomalies due to the solar flares were observed in all the paths. The SPA amplitudes in different paths vary in the range from 10 to $282^{\circ}$.

- A weak correlation between the SPA amplitudes and path lengths and the signal frequency was found.

- On average, the decrease of the reflecting layer height was about 12 and $9 \mathrm{~km}$ for the first and second event respectively. In $75 \%$ of observations the decrease was more than $7 \mathrm{~km}$.

- Spectral analysis revealed that the maximum of spectral density both for X-ray and LF signals was in the interval 2-16 min. The shapes of X-ray and LF signal spectra were very similar and differed markedly from those for undisturbed days.

Data availability. Our VLF-LF data are property of our group, and they are not publicly accessible. According to an agreement between all the participants, we cannot make the data openly accessible. Data can be provided under commercial conditions via direct request to rozhnoi@ifz.ru.

Author contributions. AR and MS led the overall research. VF and SS performed the analysis of the solar data. All authors contributed to the data analysis of the receiving stations and reviewed the paper.

Competing interests. The authors declare that they have no conflict of interest.

Special issue statement. This article is part of the special issue "Solar magnetism from interior to corona and beyond". It is a result of the Dynamic Sun II: Solar Magnetism from Interior to Corona, Siem Reap, Angkor Wat, Cambodia, 12-16 February 2018.

Acknowledgements. Viktor Fedun is grateful to The Royal Society (International Exchanges Scheme), NERC, STFC Consolidated Grants and Air Force Office of Scientific Research (USA) for support provided.
Financial support. This research has been supported by the Royal Society, International Exchanges Scheme - 2017/R1 Standard Programme (grant no. IE170301), the Science and Technology Facilities Council (STFC) (grant no. ST/M000826/), the Natural Environment Research Council (NERC) (grant no. NE/P017061/1), and the Air Force Office of Scientific Research (USA) (grant no. 18IOA009).

Review statement. This paper was edited by Manuela Temmer and reviewed by Pier Francesco Biagi and one anonymous referee.

\section{References}

Chilton, C. J., Steele, F. K., and Norton, R. B.: Very-LowFrequency Phase Observations of Solar Flare Ionization in the D Region of the Ionosphere, J. Geophys. Res., 68, 5421, https://doi.org/10.1029/JZ068i019p05421, 1963.

Deshpande, S. D., Subrahmanyam, C. V., and Mitra, A. P.: Ionospheric effects of solar flares - I. The statistical relationship between X-ray flares and SID's, J. Atmos. Terr. Phys., 34, 211-227, https://doi.org/10.1016/0021-9169(72)90165-1, 1972.

Druzhin, G. I., Mel'nikov, A. N., and Cherneva, N. V.: Manifestation of Earth's diurnal periods in VLF radiation, Doklady Earth Sciences, 457, 842-844, https://doi.org/10.1134/S1028334X14070046, 2014.

Grubor, D. P., Šulić, D. M., and Žigman, V.: Classification of $\mathrm{X}$-ray solar flares regarding their effects on the lower ionosphere electron density profile, Ann. Geophys., 26, 1731-1740, https://doi.org/10.5194/angeo-26-1731-2008, 2008.

Hayes, L. A., Gallagher, P. T., McCauley, J., Dennis, B. R., Ireland, J., and Inglis, A.: Pulsations in the Earth's Lower Ionosphere Synchronized With Solar Flare Emission, J. Geophys. Res.-Space Phys., 122, 9841-9847, https://doi.org/10.1002/2017JA024647, 2017.

Kamada, T.: Synoptic report of VLF sudden phase anomalies observed at Toyokawa, Japan, J. Geomagn. Geoelectr., 37, 667699, https://doi.org/10.5636/jgg.37.667, 1985.

Khan, I., Devi, M. I., Arunamani, T., and Madhusudhana Rao, D. N.: A synoptic study of VLF sudden phase anomalies recorded at Visakhapatnam, Earth Planets Space, 57, 1073-1081, 2005.

Kreplin, R. W., Chubb, T. A., and Friedmann, H.: X-Ray and Lyman-Alpha Emission from the Sun as Measured from the NRL SR-1 Satellite, J. Geophys. Res., 67, 2231-2253, https://doi.org/10.1029/JZ067i006p02231, 1962.

Li, N., Lei, J., Luan, X., Chen, J., Zhong, J., Wu, Q., Xu, Z., and Lin, L.: Responses of the $\mathrm{D}$ region ionosphere to solar flares revealed by MF radar measurements, J. Atmos. Solar-Terr. Phy., 182, 211 216, https://doi.org/10.1016/j.jastp.2018.11.014, 2019.

Liu, F., Qin, Z., Zhu, B., Ma, M., Chen, M., and Shen, P. Observations of ionospheric D layer fluctuations during sunrise and sunset by using time domain waveforms of lightning narrow bipolar events, Chinese J. Geophys., 61, 484-493, https://doi.org/10.6038/cjg2018K0658, 2018.

Mitra, A. P. (Ed.): Ionospheric effects of solar flares, Astrophys. Space Sc. L., 46, 307 pp., https://doi.org/10.1007/978-94-0102231-6, 1974. 
Muraoka, Y., Murata, H., and Sato, T.: The quantitative relationship between VLF phase deviations and 1-8 A solar X-ray fluxes during solar flares, J. Atmos. Terr. Phys., 39, 787-792, https://doi.org/10.1016/0021-9169(77)90140-4, 1977.

Raulin, J.-P., Bertoni, F. C. P., Gavilán, H. R., Guevara-Day, W., Rodriguez, R., Fernandez, G., Correia, E., Kaufmann, P., Pacini, A., Stekel, T. R. C., Lima, W. L. C., Schuch, N. J., Fagundes, P. R., and Hadano, R.: Solar flare detection sensitivity using the South America VLF Network (SAVNET), J. Geophys. Res.-Space Phys., 115, A07301, https://doi.org/10.1029/2009JA015154, 2010.

Raulin, J.-P., Trottet, G., Kretzschmar, M., Macotela, E. L., Pacini, A., Bertoni, F. C. P., and Dammasch, I. E.: Response of the low ionosphere to X-ray and Lyman- $\alpha$ solar flare emissions, J. Geophys. Res.-Space Phys., 118, 570-575, https://doi.org/10.1029/2012JA017916, 2013.
Thomson, N. R., Rodger, C. J., and Clilverd, M. A.: Large solar flares and their ionospheric D region enhancements, J. Geophys. Res.-Space Phys., 110, A06306, https://doi.org/10.1029/2005JA011008, 2005.

Wait, J. R. and Spices, K. P.: Characteristics of the Earth ionosphere waveguide for VLF radio waves, NBS Tech. Note, p. 300, 1964.

Westfall, W. D.: Prediction of VLF Diurnal Phase Changes and Solar Flare Effects, J. Geophys. Res., 66, 2733-2736, https://doi.org/10.1029/JZ066i009p02733, 1961.

Whitten, R. C. and Poppoff, I. G.: Physics of the Lower Ionosphere, Prentice-Hall, New York, 232 pp., 1965.

Žigman, V., Grubor, D., and Šulić, D.: D-region electron density evaluated from VLF amplitude time delay during Xray solar flares, J. Atmos. Solar-Terr. Phy., 69, 775-792, https://doi.org/10.1016/j.jastp.2007.01.012, 2007. 